\title{
SERIES CONNECTION OF RESONANT TUNNELING DIODES FOR ELIMINATING SPURIOUS OSCILLATIONS
}

\author{
Tetsu Fujii ${ }^{1.2}$, Olga Boric-Lubecke ${ }^{1}$, Jongsuck Bae ${ }^{1.2}$, and Koji Mizuno ${ }^{1.2}$ \\ ${ }^{1}$ Photodynamics Research Center, The Institute of Physical and Chemical Research, \\ 19-1399 Aza-Koeji, Nagamachi, Aoba-ku, Sendai 980, Japan \\ ${ }^{2}$ Research Institute of Electrical Communications, Tohoku University, 2-1-1 Katahira, \\ Aoba-ku, Sendai 980-77, Japan \\ kotetsu@riec.tohoku.ac.jp
}

\begin{abstract}
$\underline{\text { Abstract }}$
Removal of spurious oscillations produced by RTD oscillators, using series connection, has been investigated. It has been shown experimentally that tunnei diode oscillators with two or more diodes do not produce the unwanted low frequency oscillations, which occur in a corresponding single-diode circuit. Preliminary experiments with the series connection of two RTD's have also been carried out, demonstrating possibility to construct such oscillators.

Introduction

Resonant tunneling diode (RTD) oscillators offer significant potential for submillimeter wave generation. However, the RTD's inherently broad-band negative differential resistance usually leads to undesirable low frequency spurious oscillations which are related to bias circuitry. Several methods for suppressing spurious oscillations have been proposed so far, including lossy line stabilization [1], and on wafer stabilization with an integrated Schottky diode [2]. Unfortunately, these methods introduce additional losses, and thus decrease oscillator efficiency. Here we propose using a series combination of two RTD's to eliminate unwanted low frequency oscillations, without affecting the efficiency of the oscillator.
\end{abstract}

It has been shown experimentally that the output power of tunnel diode oscillators can be increased by connecting multiple devices in series [3]. Additionally, this seriesconnection exhibits low frequency cutoff, and a minimum oscillation amplitude, which make oscillator circuits less prone to spurious oscillations. The increased stability of such oscillators has previously been investigated theoretically [4]. Here, we will present experimental results that show the elimination of spurious oscillations through the seriesconnection of tunnel diodes, and also some preliminary results with RTD's. The effect of differing I-V characteristics between two series connected diodes will be discussed as well.

\section{Series Connection}

When two identical diodes, which exhibit a negative differential resistance (NDR) region in their DC I-V curves, are connected in series and biased with a single battery with sufficient voltage to have bias both in the NDR region, there are three possibilities for the bias voltage distribution (Fig. 1): both diodes can be biased in the NDR region and have the same DC voltage (solution I), or both diodes can be biased in the positive differential resistance (PDR) region, one at a voltage lower than the peak voltage $V_{p}$, and the other at a voltage higher than the valley voltage $V_{v}$ (solutions II and III). If there is no RF signal present in the circuit, solution I is unstable, and will move towards solutions II 
or III in a very short time. This phenomenon is due to the nature of the NDR, and has been previously described as the DC instability [4]. It is analogous to the case of a single tunneling diode biased with the constant current source: the device always seeks the stable alternative, which is a bias outside of the NDR region. When an RF signal is present across the series connection, solution I might be stable if oscillation amplitude $\left(\mathrm{V}_{\mathrm{rf}}\right)$ and frequency are above the minimum values $\left(\mathrm{V}_{\mathrm{rfmin}}, \mathrm{f}_{\mathrm{min}}\right)$, which can be calculated from the device parameters [4].

If the diode I-V curves are not identical (Fig. 2), the lower current diode (diode 1) cannot achieve the peak current $I_{p}$ of the higher current diode (diode 2) at the voltage $V_{p}$ (Fig.). Initially, diode 1 will cross the peak current $I_{p 1}$ and enter the NDR region. Only when $V_{d 1}$ exceeds the valley voltage $V_{v}$, can diode 2 cross the peak current $I_{p 2}$ and become biased in the NDR region. As a result of either DC instability or difference in DC I-V curves, the series connection DC I-V curve will exhibit multiple peaks (Fig 5a, 6a) if there is no oscillation. When an RF signal is present, it is possible to bring both bias points in the NDR region, and achieve stable oscillation. However, similar to the case of identical diodes, certain oscillation amplitude and frequency constraints must be satisfied. Displacement currents through the parasitic capacitance of the device must "compensate" for the difference between the conductive current at the current peak $I_{\text {peak }}$, and from this requirement a simple way to estimate minimum oscillation frequency $f_{\min }$ can be deduced:

$$
\mathrm{f}_{\min }=\Delta \mathrm{I}_{\text {peak }} / 2 \pi \Delta \mathrm{V}_{\mathrm{rf}} \mathrm{C} \text {, }
$$

where $\mathrm{C}$ is the capacitance of a single diode, and $\mathrm{V}_{\mathrm{rf}}$ the difference between diode oscillation amplitudes. Since $\mathrm{V}_{\mathrm{rf}}$ must be above the minimum value $\mathrm{V}_{\mathrm{rfmin}}$, determined by the DC instability, we must assure $V_{r f}$ is sufficiently small to keep this condition satisfied. A reasonable estimate is that $\mathrm{V}_{\mathrm{rf}}$ should be less than $10 \%$ of the voltage amplitude of one diode. If the following condition is satisfied at the oscillation frequency f,

$$
\Delta \mathrm{I}_{\text {peak }} \ll 2 \pi f \mathrm{~V}_{\mathrm{rf}} \mathrm{C}
$$

the previously described DC instability will be the prevailing phenomena for determining $f_{\min }$.

Single RTD low frequency spurious oscillations, usually associated with a resonance in bias circuitry, occur due to the absence of a low frequency cutoff $f_{\min }$, and a broad range of device impedances for which oscillation condition can be satisfied. In the case of the series connection, minimum oscillation frequency and amplitude impose further constraints on the oscillation condition, and therefore unwanted oscillations, even though theoretically possible, are not very likely to occur.

\section{Tunnel Diode Experimental Results}

Oscillators with series connected tunnel diodes have been successfully demonstrated in the past $[3,4]$. Recently, such circuits have been revisited to examine oscillator stability in more detail. The tunnel diodes and circuit topology were the same as described in $[3,4]$. These are very low current diodes, with a peak current of about 0.5 $\mathrm{mA}$, and the $\mathrm{I}-\mathrm{V}$ curves for different diodes vary by less than $10 \%$. At the $2 \mathrm{GHz}$ design frequency, equation (2) is satisfied, which means that the DC instability is the prevailing reason for the existence of $f_{\min }$ and $V_{\text {rfmin }}$, which were estimated to be $20 \mathrm{MHz}$ and 0.125 $\mathrm{V}$ respectively [4].

Low NDR current results in a small negative differential conductance, and therefore a somewhat limited impedance range for which oscillation condition can be 
satisfied, even in the case of a single diode oscillator. Still, most one diode circuits exhibited spurious oscillations to some degree. Typically, such oscillations occurred for the bias voltage near $V_{p}$, and gradually disappeared as bias voltage was increased closer to the $V_{v}$. On the other hand, while one diode spurious oscillations were also observed in multiple diode circuits for similar bias conditions, such oscillations ceased once all diodes were biased in the NDR region, and a clean signal appeared at the design frequency of 2 $\mathrm{GHz}$. Fig. 3a shows the spectrum of a single diode oscillator, for a bias voltage range of 0.07-0.09 V. Even though there is a signal at $2 \mathrm{GHz}$, a low frequency oscillation occurs as well, and produces higher harmonics and mixing components close to the design frequency. Two (and more) diode oscillators always produced only a single line (Fig. $3 b)$, which clearly indicated that series connection can effectively suppress the unwanted oscillations.

\section{Series Connection of RTD's}

Mesa type GaAs/AlAs RTD's, with the doping profile described in [5], were also used for the experiments. Mesas were formed using two types of dry etching: reactive ion etching (RIE) in a $\mathrm{PCl}_{3}$ environment, and electron cyclotron resonance reactive beam ion etching (ECR-RIBE) in a $\mathrm{Cl}_{2}$ environment. It was found that $\mathrm{ECR}$ etching produced better quality diodes, with straight mesa side walls, and twice as high peak-to-valley current ratio (about 4).

Previously, millimeter wave oscillations were achieved with these RTD's [5] in a quasi-optical resonator, using $9 \mu \mathrm{m}$ diameter mesas, and very short whisker wires. However, it would be very difficult to create a series connection for two diodes in this configuration, without integrating them monolithically. Therefore, microstrip oscillator circuits were designed using the procedure described in [3]. Diodes were contacted with whisker wires attached to the microstrip. This resulted in fairly long whiskers, about 1$1.5 \mathrm{~mm}$ long, and due to the corresponding inductance, the oscillation frequency range was limited to under $20 \mathrm{GHz}$. Since it was anticipated that an external RF source would be necessary for triggering oscillation [3], an oscillation frequency was chosen in the range of $2-5 \mathrm{GHz}$, to be compatible with the available circulators and signal sources.

Initially, a "back-to-back" connection of two RTD's was attempted (Fig. 4a). An RTD chip was mounted on a simple microstrip circuit, and diode contacts were made with two whisker wires attached to the microstrip. While such a configuration offered easy assembly and small distance between the diodes, individual diode I-V curves were quite different (Fig. 4b), and it was not possible to achieve simultaneous oscillation. A small difference in the width of the spacer layer on the mesa and substrate sides of the double barriers may partially account for this variation in I-V curves. Symmetric doping profile wafers are being processed to check the influence of this asymmetry. However, physical asymmetry of the diode orientation could also contribute to the difference in I-V curves. It might be possible to overcome this problem by etching most of the highly doped GaAs substrate in between the mesas.

Next, two chips were connected in cascade (Fig. 5a), and diode contacts were repeated until it was assured that the best uniformity in the I-V curves was achieved. The DC I-V curve for the series connection was measured while individual diode voltages were monitored using a small whisker probe in between the chips (Fig. 5b). The diodes were then biased with sufficient voltage to bring them both to the middle of the NDR region, and an external RF signal was applied through the circulator to switch the bias points from the PDR regions to the NDR region. It was possible to have both diodes biased in the NDR region while the external signal was present (Fig. 6), and diodes were clearly oscillating. These results indicate that RTD DC instability may be removed using 
an external source for a short amount of time, similarly to the case of tunnel diodes [3]. However, after the external RF signal was turned off, the bias points switched back to the PDR region and there was no oscillation. Careful examination of the I-V curves showed that even though the diode difference appeared very small (less than $5 \%$ ), it was still significant according to equations 1 and 2, due to the very small device capacitance (about $80 \mathrm{fF}$ ), and thus possibly responsible for the absence of oscillations. Shunting the diodes with capacitors of about $1 \mathrm{pF}$ might help to solve this problem, and we are currently investigating oscillators with shunt capacitors.

\section{Conclusions}

Benefits of the series connection of tunneling diodes for the removal of spurious oscillations have been discussed. It was demonstrated experimentally that the series connection of tunnel diodes has improved performance over a single diode oscillator. Series connection of RTD's has also been investigated. So far, we have experimentally demonstrated RF switching of the bias points to the NDR region in the case of two series connected RTD's, and are presently working on the further oscillator development.

\section{Acknowledgments}

We would like to thank $H$. Sakaki, T. Noda, and $M$. Narihiro from the University of Tokyo for supplying MBE grown GaAs wafers. Part of this work was carried out at the Laboratory for Electronic Intelligent Systems, Research Institute of Electrical Communications, Tohoku University, supported by a Grant-in Aid of Scientific Research from the Ministry of Education, Science and Culture of Japan.

\section{References}

[1] K. D. Stefan and S. C. Wong, "Lossy-Line Stabilization of Negative-Resistance Diodes for Integrated-Circuit Oscillators," Proc. of the Second Intemational Symposium on Space Terahertz Technology, pp. 154-162, February 26-28, 1991.

[2] M. Reddy, R. Y. Yu, H. Kroemer, M. J. W. Rodwell, S. C. Martin, R. E. Muller, and R. P. Smith, "Bias Stabilization for Resonant Tunnel Diode Oscillators," IEEE Microwave and Guided Wave Letters, vol. 5, pp. 129-221, July 1995.

[3] O. Boric-Lubecke, D. S. Pan and T. Itoh, "Fundamental and Subharmonic Excitation of an Oscillator with Several Tunneling Diodes in Series", IEEE Trans. on Microwave Theory Tech., vol. 43, no. 4, pp. 969-976, April 1995.

[4] O. Boric-Lubecke, D. S. Pan and T. Itoh, "DC Instability of the Series Connection of Tunneling Diodes" , IEEE Trans. on Microwave Theory Tech., vol. 44, no. 6, pp. 936-943, June 1996.

[5] T. Fujii, H. Mazaki, F. Takei, J. Bae, M Narihiro, T. Noda, H. Sasaki, and K. Mizuno, "Coherent Power combining of Millimeter Wave Resonant Tunneling Diodes in a Quasi-Optical Resonator," 1996 IEEE MTT-S Digest, pp. 919-922. 


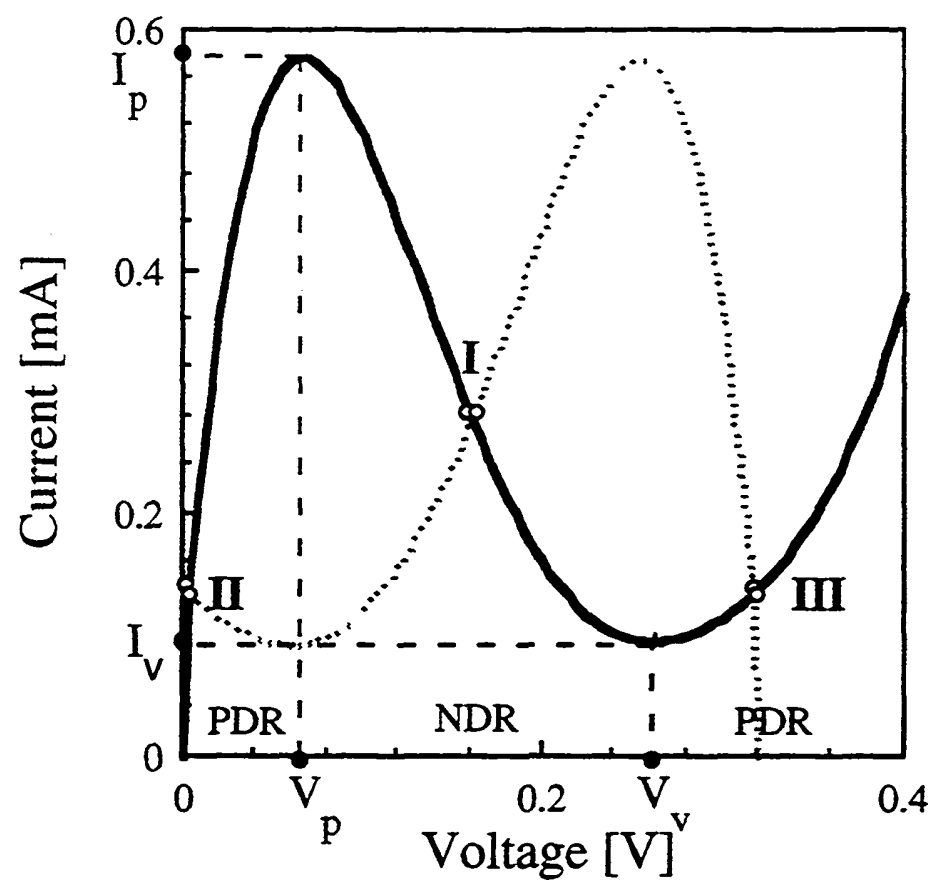

Fig. 1 Three solutions for the bias voltage distribution of tunneling diodes: both diodes can be biased in the NDR region (I), or in the PDR region, one at the voltage lower than the peak voltage $V_{p}$, and the other at the voltage higher than the valley voltage $V_{v}$ (II and III).

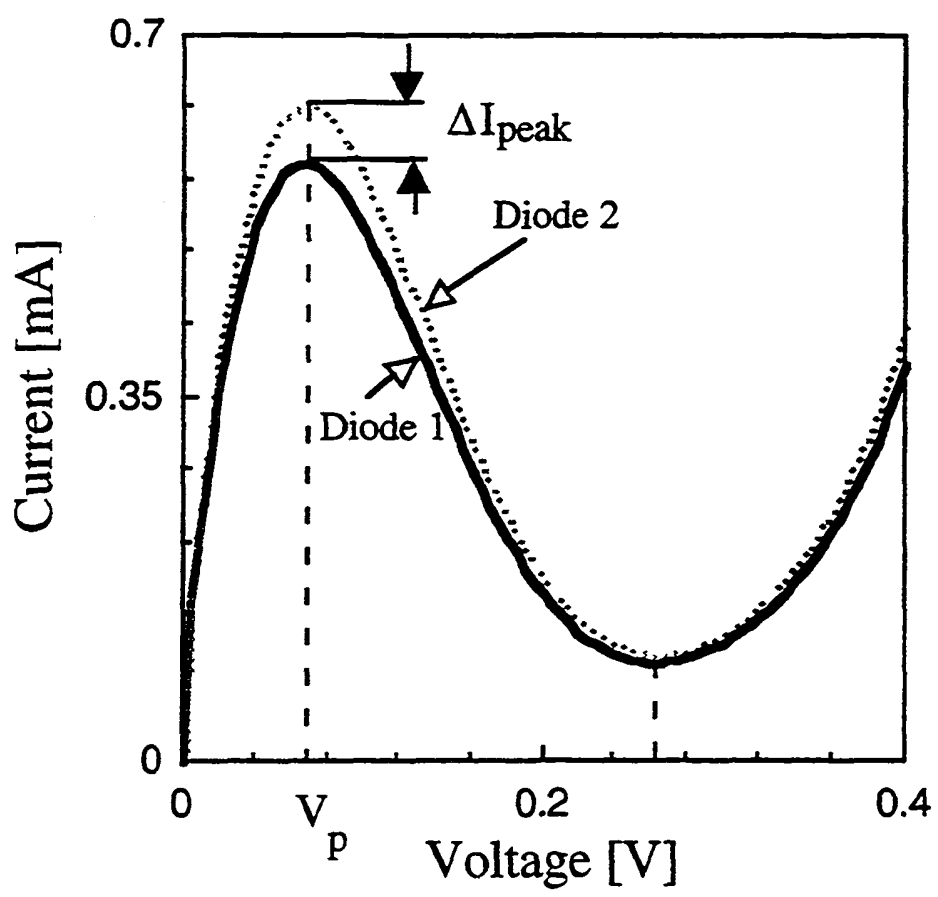

Fig. 2 If the diode I-V curves are not identical, a lower current diode (diode 1) cannot achieve the peak current $I_{p}$ of the higher current diode (diode 2) at the voltage $V_{p}$. 


\section{ATTEN 10dB MKR -36.67dBm}

RL $-10.0 \mathrm{dBm} 10 \mathrm{~dB} /$ div. $\quad 2.020 \mathrm{GHz}$

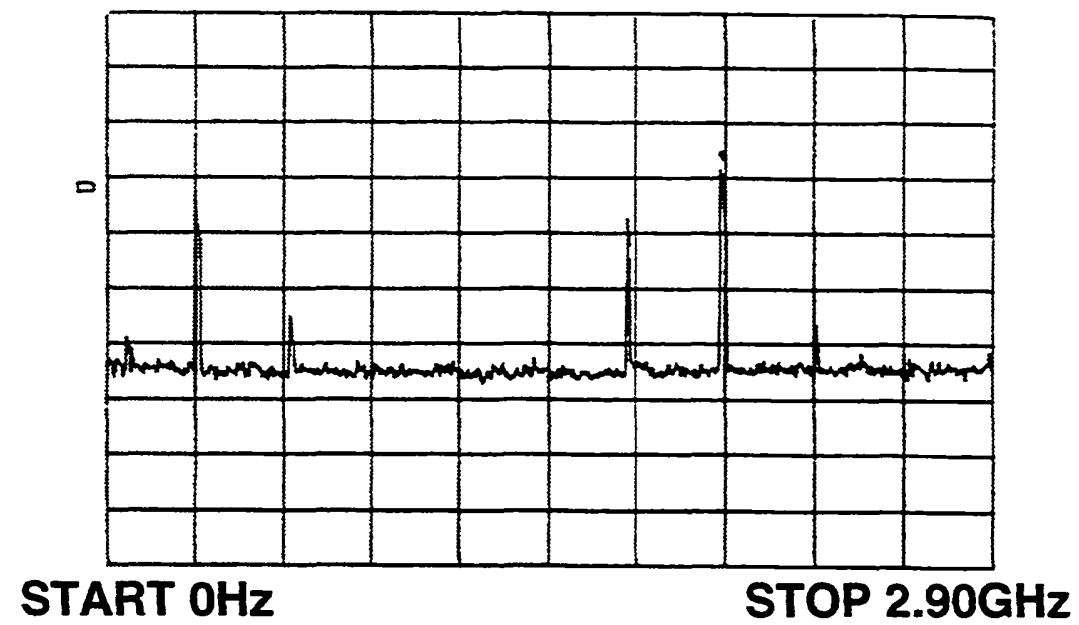

(a)

\section{ATTEN 10dB \\ MKR -32.67dBm \\ RL $-10.0 \mathrm{dBm} 10 \mathrm{~dB} /$ div. $\quad 2.011 \mathrm{GHz}$}

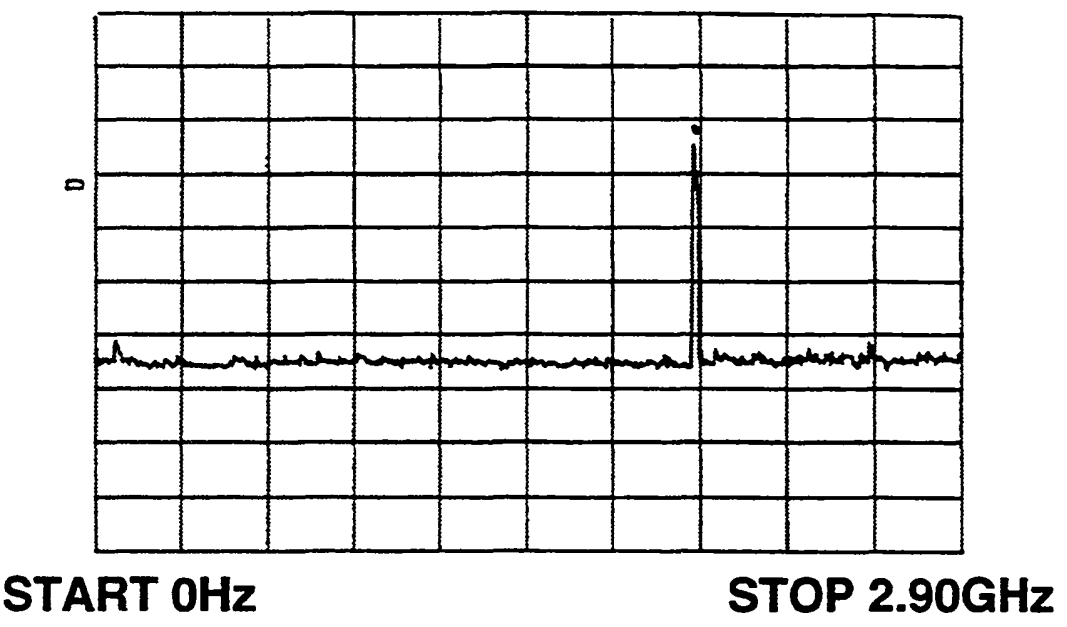

(b)

Fig. 3 Output spectrum of one diode oscillator (a), and a two diode oscillator (b). One diode oscillator produces spurious components, whereas two diode circuit oscillates only at the design frequency of $2 \mathrm{GHz}$. 


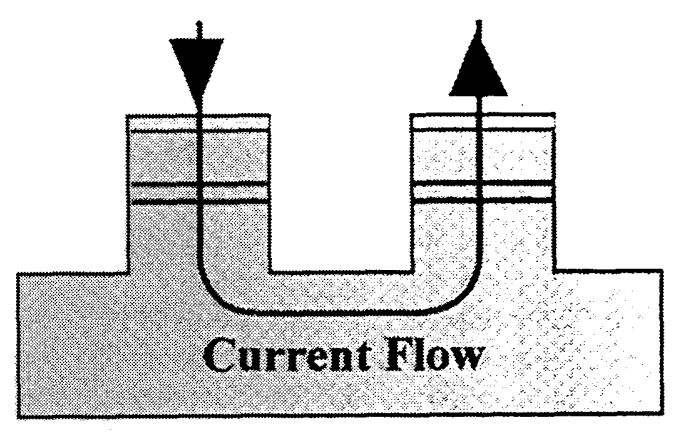

(a)

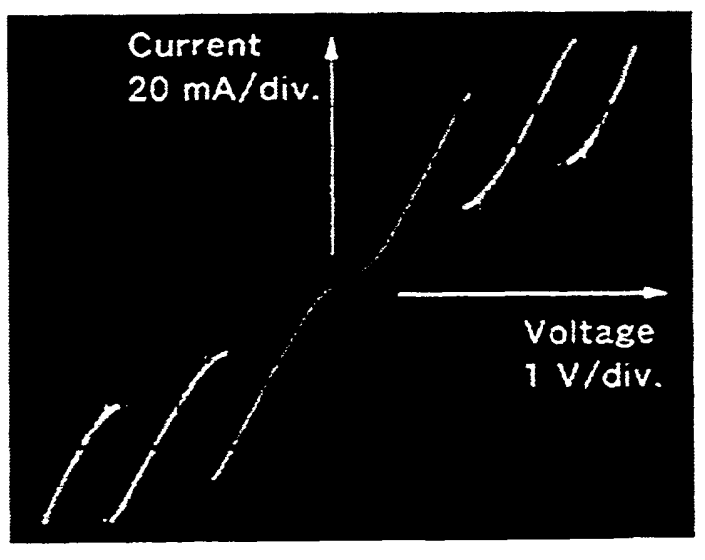

(b)

Fig. 4 Schematic of a "back-to-back" configuration (a), and measured DC I-V curve (b). Due to the large difference in peak current. it was not possible to construct an oscillator with this configuration.

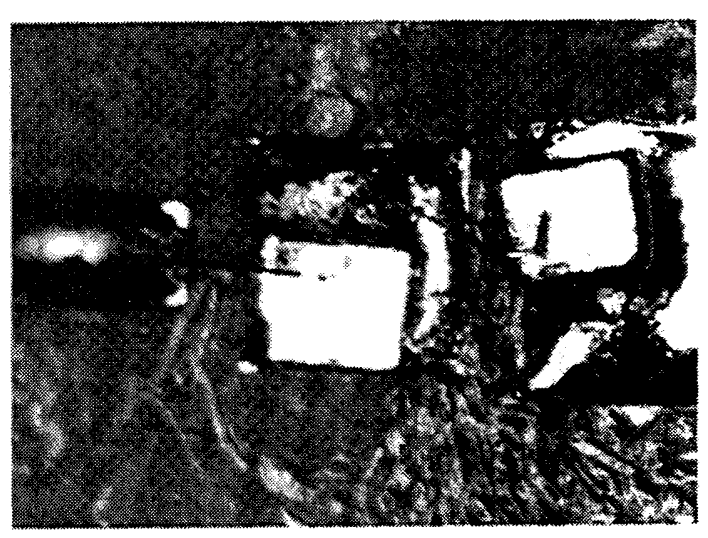

(a)

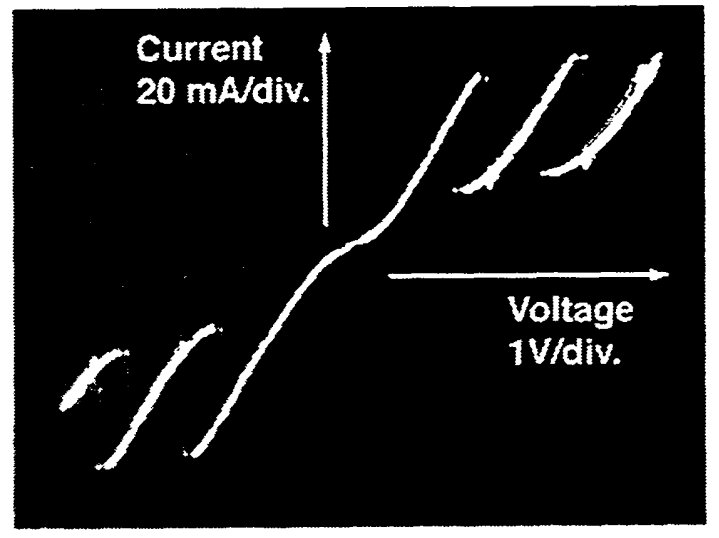

(b)

Fig. 5 Photograph of the microstrip cascade connection (a), and measured DC I-V curve (b). Very good uniformity of individual diodes I-V's can be achieved in this case. 


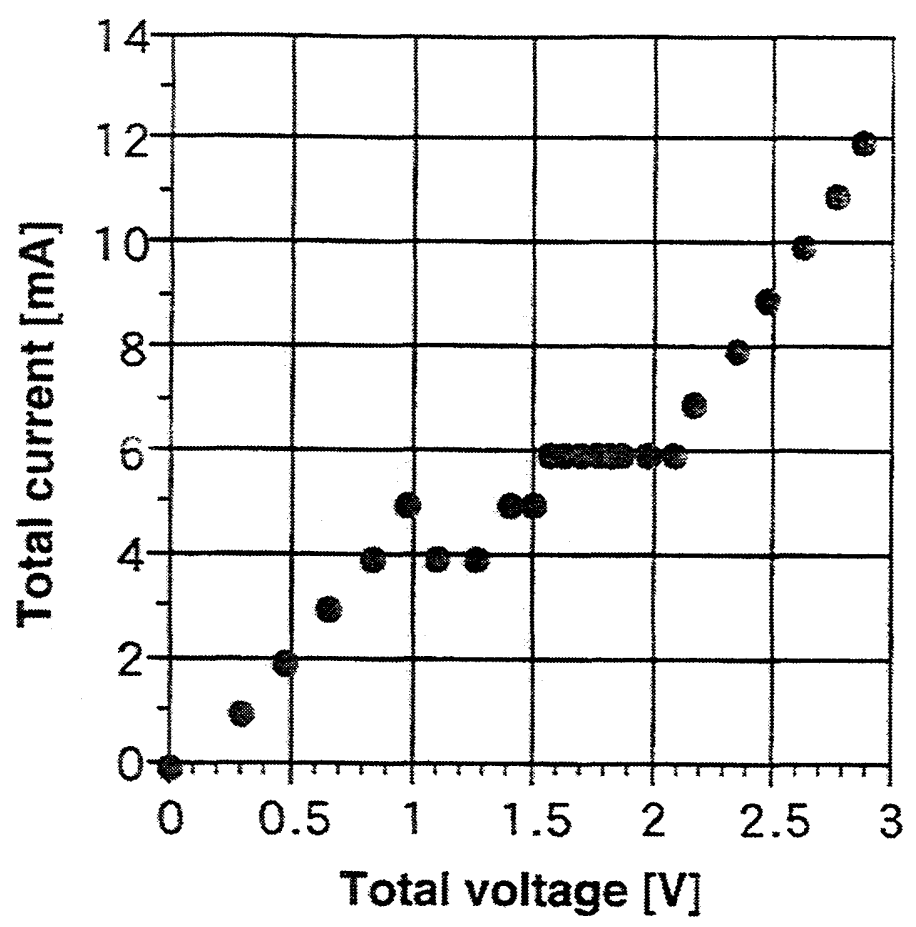

(a)

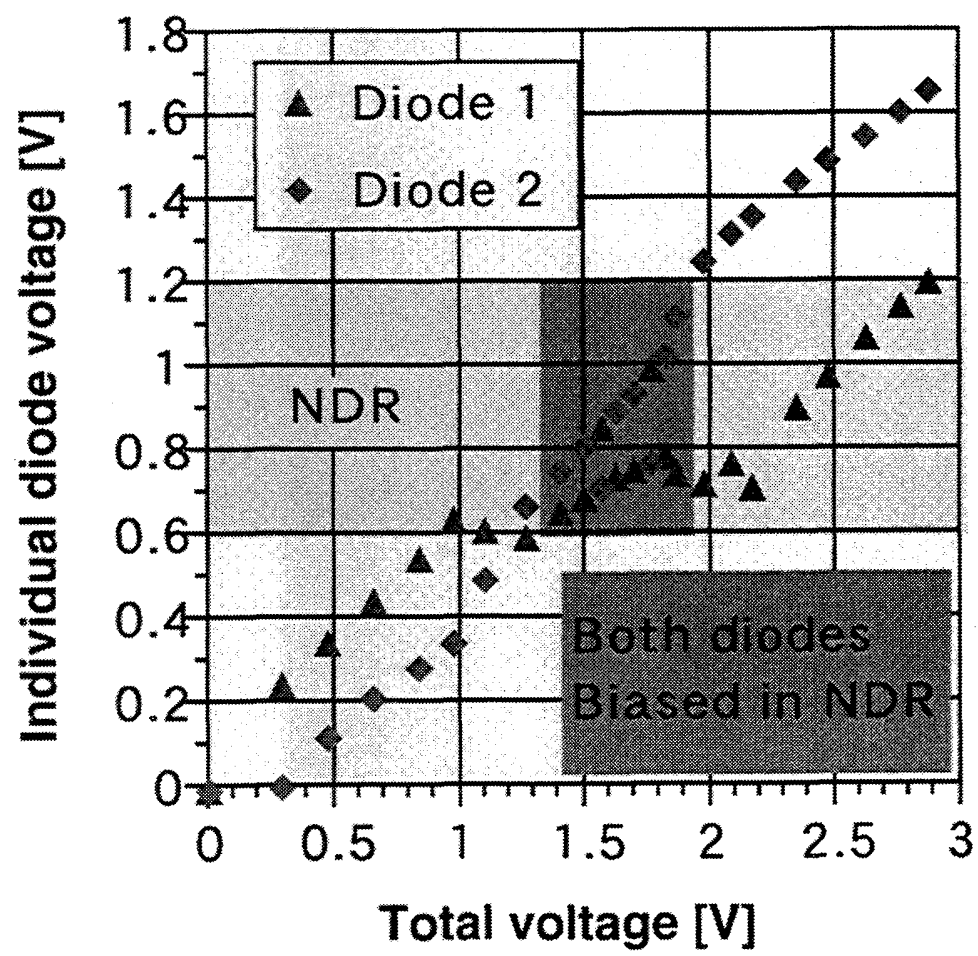

(b)

Fig. 6 DC I-V curve of a series connection during RF triggering (a), and the individual between diode voltages (b). Both RTD's can be biased simultaneously in the NDR region. 